\title{
Ramond-Ramond Central Charges in the Supersymmetry Algebra of the Superstring
}

\author{
Nathan Berkovits* \\ Instituto de Física Teórica, Universidade Estadual Paulista, Rua Pamplona 145, São Paulo SP 01405-900, Brasil
}

(Received 12 June 1997)

\begin{abstract}
The free action for the massless sector of the type II superstring was recently constructed using closed Ramond-Neveo-Schwarz superstring field theory. The supersymmetry transformations of this action are shown to satisfy an $N=2 D=10$ supersymmetry algebra with Ramond-Ramond central charges. [S0031-9007(97)03906-9]

PACS numbers: 11.25.-w
\end{abstract}

One of the most important reasons for believing superstring theory is related to a higher-dimensional theory is the fact that nonperturbative superstring states called D-branes carry Ramond-Ramond (R-R) charge [1]. This provides a connection between eleven-dimensional Kaluza-Klein states and superstring theory since RamondRamond charge is related to momentum in the eleventh direction. In this Letter, it is shown that R-R central charges are present in the supersymmetry algebra of the superstring, providing an even more direct connection with a higher-dimensional theory.

Ramond-Ramond central charges are usually assumed to be absent in the spacetime-supersymmetry algebra of the superstring. This assumption is based on three arguments: (i) No Perturbative superstring states carry RamondRamond charge. (ii) The left- and right-moving spacetimesupersymmetry generators anticommute with each other in the RNS formalism. (The left-moving with left-moving SUSY generators anticommute to give the usual momentum and NS-NS one-brane charge, but no NS-NS five-brane charge [2].) (iii) There is no Wess-Zumino term for Ramond-Ramond fields in the standard GreenSchwarz (GS) sigma model [3].

Although all of these arguments are correct, it will be shown here that R-R central charges are nevertheless present in the supersymmetry (SUSY) algebra of the superstring. Because of the first argument, these charges can be detected only through the Abelian transformation of the R-R gauge field. Note that when the "picture" is treated properly [4], zero-momentum R-R gauge fields are present in the string field, although they decouple from all perturbative superstring states. Using superstring field theory, the transformation of the R-R gauge field is enough to construct the global R-R charge out of RNS matter and ghost world sheet variables. When acting on perturbative superstring states, this charge is BRST trivial, which is not ruled out by the second argument since the supersymmetry algebra in the RNS description closes only up to gauge transformations and equations of motion. Finally, the dependence of the R-R charge on world sheet ghosts explains why the third argument is inapplicable. In the standard GS sigma model, world sheet ghosts are not understood, as can be seen by the absence of a Fradkin-Tseytlin term [5] which should couple the spacetime dilaton to world sheet curvature.

Because of picture-changing difficulties, a free action for the R-R fields of the type II superstring has only recently been constructed [4]. In the massless sector, the R-R string field depends on an "electric" gauge field $A_{(+)}^{\alpha \beta}$, a "magnetic" gauge field $A_{(-)}^{\alpha \beta}$, and an infinite set of auxiliary fields $F_{(n)}^{\alpha \beta}$ for $n=1$ to $\infty$. [ $\alpha$ and $\beta$ are 16-component $\mathrm{SO}(9,1)$ spinor indices whose chirality depends on whether one is discussing type IIA or Type IIB.] This set of massless fields can be understood using the usual RNS formalism if the R-R vacuum state is chosen to be annihilated by $\gamma_{L}^{0}$ and $\beta_{R}^{0}$ (as opposed to $\beta_{L}^{0}$ and $\left.\beta_{R}^{0}\right)$, where $\left[\beta_{L}^{0}, \gamma_{L}^{0}\right]$ and $\left[\beta_{R}^{0}, \gamma_{R}^{0}\right]$ are the zero modes of the left- and right-moving bosonic ghosts.

After adding the NS-R, R-NS, and NS-NS massless sectors, the action is invariant under coordinate and $b_{\mu \nu}$ gauge transformations, under local $N=2 D=10$ supersymmetry transformations, and under R-R gauge transformations of $A_{(+)}^{\alpha \beta}$ and $A_{(-)}^{\alpha \beta}$. It will be shown here that the commutator of two supersymmetry transformations gives a coordinate transformation, a $b_{\mu \nu}$ gauge transformation, and a R-R gauge transformation. This means that the SUSY algebra of the type II superstring contains R-R central charges as well as the usual NS-NS one-brane central charge.

Note that even without knowing superstring field theory, the presence of Ramond-Ramond central charges could have been predicted based on the role of the dilaton as a conformal compensator [6]. In $N=2 D=4$ supergravity coupled to vector multiplets, it is well known that the $N=2 D=4$ SUSY algebra contains central charges of the form [7]

$$
\left[\delta_{q_{i}^{\alpha}}\left(u_{i}^{\alpha}\right), \delta_{q_{j}^{\beta}}\left(v_{j}^{\beta}\right)\right]=\sum_{n} \delta_{C_{(n)}}\left(W_{(n)} u^{i \alpha} v_{\alpha}^{j} \epsilon_{i j}\right),
$$

where $i$ is an $\mathrm{SU}(2)$ index, $W_{(n)}$ is the expectation value of the complex scalar in the $n$th vector multiplet, $\delta_{q_{i}^{\alpha}}\left(u_{i}^{\alpha}\right)$ is the SUSY transformation with parameter $u_{i}^{\alpha}$, and $\delta_{C_{(n)}}\left(\rho_{(n)}\right)$ is the gauge transformation of the $n$th gauge field with parameter $\rho_{(n)}$.

One of these vector multiplets is the vector compensator multiplet and, as shown in [6], the expectation value of the 
scalar in this multiplet is $\left\langle e^{-\phi}\right\rangle=g^{-1}$, where $\phi$ is the dilaton and $g$ is the string coupling constant. So one expects R-R central charges in the type II superstring, at least after compactifying to four dimensions. This suggests that a proper understanding of conformal compensators in ten dimensions will help in understanding the role of R-R central charges.

Superstring field theory (the massless $R-R$ sector).The massless R-R contribution to the free type II superstring action was recently constructed using closed superstring field theory [4]. Although "nonminimal" fields [8] were needed for this construction, the equations of motion and gauge invariances can be easily analyzed using the standard RNS world sheet variables.

Physical closed superstring states are described by fields $|\Phi\rangle$ of zero total ghost number where total ghost number is $g_{L}+g_{R}-2, \quad g_{L / R}=\oint d \sigma\left(b_{L / R} c_{L / R}-\right.$ $\left.\eta_{L / R} \xi_{L / R}\right)$, and the bosonic ghosts have been fermionized as $\beta=\partial \xi e^{-\phi}$ and $\gamma=\eta \epsilon^{\phi}$. (The -2 is present so that physical states carry zero ghost number, implying that the SL(2)-invariant vacuum carries a -2 total ghost number.) Note that this definition of ghost number is slightly modified from the standard one, $g=\oint d \sigma(b c-\partial \phi)$, although they agree at zero picture. [Picture is defined by $P=\oint d \sigma(\eta \xi-\partial \phi)$.] The modified definition of $g$ is necessary in order that the spacetime-supersymmetry generators carry zero ghost number.

These string fields must satisfy the constraints $b_{-}^{0} \times$ $|\Phi\rangle=L_{-}^{0}|\Phi\rangle=\eta_{L / R}^{0}|\Phi\rangle=0$ and are defined up to the gauge transformation $\delta|\Phi\rangle=Q|\Lambda\rangle$, where $Q=Q_{L}+$ $Q_{R}$ is the BRST charge, $b_{-}^{0}=b_{L}^{0}-b_{R}^{0}, L_{-}^{0}=L_{L}^{0}-$ $L_{R}^{0}$ is the difference of the left- and right-moving energies, respectively, $\eta_{L / R}^{0}|\Phi\rangle=0$ implies no dependence on $\xi_{L / R}^{0}$, and $|\Lambda\rangle$ is a gauge field of -1 total ghost number satisfying $L_{-}^{0}|\Lambda\rangle=b_{-}^{0}|\Lambda\rangle=\eta_{L / R}^{0}|\Lambda\rangle=0$. The equation of motion is $Q|\Phi\rangle=0$.

Finally, there is a constraint coming from the restriction to a single picture. Surprisingly, different choices of leftand right-moving pictures $P_{L}$ and $P_{R}$ give different sets of on-shell fields. (This occurs because the inverse-picturechanging operator, $y=c \partial \xi e^{-2 \phi}$ does not commute with $b_{-}^{0}$.) For example, it will be found that there are onshell constant modes in the R-R sector if $\left(P_{L}, P_{R}\right)=$ $\left(-\frac{3}{2},-\frac{1}{2}\right)$, but not if $\left(P_{L}, P_{R}\right)=\left(-\frac{1}{2},-\frac{1}{2}\right)$. Since one wants to reproduce the equations of motion from Ref. [4] where on-shell zero-momentum R-R fields were found using an action principle, $\left(P_{L}, P_{R}\right)$ will be chosen to be $\left(-\frac{3}{2},-\frac{1}{2}\right)$ in the R-R sector.

This means that R-R states can be constructed using nonnegative modes acting on a vacuum $|0\rangle_{\mathrm{R}-\mathrm{R}}^{\alpha \beta}$ which satisfies $b_{L}^{0}|0\rangle_{\mathrm{R}-\mathrm{R}}^{\alpha \beta}=b_{R}^{0}|0\rangle_{\mathrm{R}-\mathrm{R}}^{\alpha \beta}=\gamma_{L}^{0}|0\rangle_{\mathrm{R}-\mathrm{R}}^{\alpha \beta}=\beta_{R}^{0}|0\rangle_{\mathrm{R}-\mathrm{R}}^{\alpha \beta}=0$.

[The picture $\left(P_{L}, P_{R}\right)=\left(-\frac{1}{2},-\frac{1}{2}\right)$ would correspond to a vacuum satisfying $\beta_{L}^{0}|0\rangle_{\mathrm{R}-\mathrm{R}}^{\alpha \beta}=\beta_{R}^{0}|0\rangle_{\mathrm{R}-\mathrm{R}}^{\alpha \beta}=0$.] In terms of the $\mathrm{SL}(2)$-invariant vacuum, $|0\rangle_{\mathrm{R}-\mathrm{R}}^{\alpha \beta}$ is $c_{L} \times$ $e^{-\frac{3}{2} \phi_{L}} \sum_{L}^{\alpha} c_{R} e^{-\frac{1}{2} \phi_{R}} \sum_{R}^{\beta}$, where $\sum_{L / R}^{\alpha}$ is the left- and right- moving spin field of weight $\frac{5}{8}$. The $\psi_{L}^{\mu}$ and $\psi_{R}^{\mu}$ zero modes are treated like $\mathrm{SO}(9,1)$ gamma matrices which transform the bispinor indices on $|0\rangle_{\mathrm{R}-\mathrm{R}}^{\alpha \beta}$. Furthermore, all string states must be GSO projected in the usual way.

Constructing all possible states of zero ghost number, the massless R-R states of the type II superstring are given by

$$
\begin{aligned}
|\Phi\rangle_{\mathrm{R}-\mathrm{R}}=\sum_{n=0}^{\infty} & {\left[A_{(n)}^{\alpha \beta}(x)\left(\beta_{L}^{0}\right)^{n}\left(\gamma_{R}^{0}\right)^{n}\right.} \\
& \left.+F_{(n)}^{\alpha \beta}(x) c_{+}^{0}\left(\beta_{L}^{0}\right)^{n+1}\left(\gamma_{R}^{0}\right)^{n}\right]|0\rangle_{\mathrm{R}-\mathrm{R}}^{\alpha \beta},
\end{aligned}
$$

where $c_{+}^{0}=c_{L}^{0}+c_{R}^{0}$. Note that the GSO projection implies that the bispinor indices on $A_{(n)}^{\alpha \beta}$ and $F_{(n)}^{\alpha \beta}$ have different $\mathrm{SO}(9,1)$ chirality if $n$ is even or odd. [In the above expressions, contracted spinor indices always have opposite $\mathrm{SO}(9,1)$ chiralities.]

Using the gauge string field,

$$
|\Lambda\rangle_{\mathrm{R}-\mathrm{R}}=\sum_{n=2}^{\infty} \Lambda_{(n)}^{\alpha \beta}(x) c_{+}^{0}\left(\beta_{L}^{0}\right)^{n}\left(\gamma_{R}^{0}\right)^{n-2}|0\rangle_{\mathrm{R}-\mathrm{R}}^{\alpha \beta},
$$

it is easy to check that $A_{(n)}^{\alpha \beta}$ can be algebraically gauged away for $n>1$. The equation of motion $Q|\Phi\rangle=0$, together with the requirement that only a finite number of fields are nonzero (this requirement can be interpreted as a normalization condition on the string field [4]), implies that the remaining fields satisfy [4]

$$
\begin{gathered}
\hat{F}_{(0)}^{\alpha \beta}=\partial^{\alpha \gamma} A_{(0)}^{\gamma \beta}-\partial^{\beta \gamma} A_{(1)}^{\alpha \gamma}, \\
\partial^{\beta \gamma} A_{(0)}^{\alpha \gamma}-\partial^{\alpha \gamma} A_{(1)}^{\gamma \beta}=0, \\
\partial^{\alpha \gamma} \hat{F}_{(0)}^{\gamma \beta}=\partial^{\beta \gamma} \hat{F}_{(0)}^{\alpha \gamma}=0, \quad F_{(2 n+1)}^{\alpha \beta}=F_{(2 n+2)}^{\alpha \beta}=0,
\end{gathered}
$$

where $\hat{F}_{(0)}^{\alpha \beta}=F_{(0)}^{\alpha \beta}+\partial^{\alpha \gamma} A_{(0)}^{\gamma \beta}$ and $\partial^{\alpha \beta}=\Gamma_{\mu}^{\alpha \beta} \partial^{\mu}$. The fields and equations of (5) are the same as those of Ref. [4] and correspond to the Bianchi identities and equations of motion for a zero-form, two-form, and four-form field strength (for type IIA) or a one-form, three-form, and selfdual five-form field strength (for type IIB).

The R-R fields $A_{( \pm)}^{\alpha \beta}=A_{(0)}^{\alpha \beta} \pm A_{(1)}^{\alpha \beta}$ play the role of electric and magnetic gauge fields with the gauge transformations

$$
\begin{aligned}
& \delta A_{(+)}^{\alpha \beta}=\partial^{\alpha \gamma} \rho_{(+)}^{\gamma \beta}+\partial^{\beta \gamma} \rho_{(+)}^{\alpha \gamma} \\
& \delta A_{(-)}^{\alpha \beta}=\partial^{\alpha \gamma} \rho_{(-)}^{\gamma \beta}-\partial^{\beta \gamma} \rho_{(-)}^{\alpha \gamma},
\end{aligned}
$$

described by the gauge string field

$$
\begin{aligned}
& |\Lambda\rangle_{\mathrm{R}-\mathrm{R}}=\left[\rho_{(0)}^{\alpha \beta}(x) \beta_{L}^{0}+\rho_{(1)}^{\alpha \beta}(x)\left(\beta_{L}^{0}\right)^{2} \gamma_{R}^{0}\right. \\
& \left.-\partial^{\beta \gamma} \rho_{(1)}^{\alpha \gamma}(x) c_{+}^{0}\left(\beta_{L}^{0}\right)^{2}\right]|0\rangle_{\mathrm{R}-\mathrm{R}}^{\alpha \beta},
\end{aligned}
$$

where $\rho_{( \pm)}^{\alpha \beta}=\rho_{(0)}^{\alpha \beta} \pm \rho_{(1)}^{\alpha \beta}$. The constant modes of $A_{( \pm)}^{\alpha \beta}$ are in the BRST cohomology since $|0\rangle_{\mathrm{R}-\mathrm{R}}^{\alpha \beta}$ and $\beta_{L}^{0} \gamma_{R}^{0}|0\rangle_{\mathrm{R}-\mathrm{R}}^{\alpha \bar{\beta}}$ cannot be written as $Q|\Lambda\rangle$ if $|\Lambda\rangle$ is restricted to contain a finite number of terms. (If an infinite number of terms allowed, $|0\rangle_{\mathrm{R}-\mathrm{R}}^{\alpha \beta}=Q \sum_{\alpha \beta}^{\infty}{ }_{n=0}^{\infty}(-1)^{n} c_{+}^{0} \times$ $\left(\beta_{L}^{0}\right)^{2+2 n}\left(\gamma_{R}^{0}\right)^{2 n}|0\rangle_{\mathrm{R}-\mathrm{R}}^{\alpha \beta} \quad$ and $\quad \beta_{L}^{0} \gamma_{R}^{0}|0\rangle_{\mathrm{R}-\mathrm{R}}^{\alpha \beta}=Q \sum_{n=0}^{\infty} \times$ $(-1)^{n} c_{+}^{0}\left(\beta_{L}^{0}\right)^{3+2 n}\left(\gamma_{R}^{0}\right)^{1+2 n}|0\rangle_{\mathrm{R}-\mathrm{R}}^{\alpha \beta}$.) One way to see the 
necessity of this restriction on $|\Lambda\rangle$ is to note that $\langle D \mid 0\rangle_{\mathrm{R}-\mathrm{R}}^{\alpha \beta}$ is nonzero where $\langle D|$ is the $D$-brane boundary state [9]. Since $\langle D|Q=0,| 0\rangle_{\mathrm{R}-\mathrm{R}}^{\alpha \beta}$ cannot be BRST trivial [10].

Note that these constant modes of $A_{( \pm)}^{\alpha \beta}$ would not be present if one had instead chosen the R-R vacuum to be annihilated by $\beta_{L}^{0}$ and $\beta_{R}^{0}$. In such a picture, the only massless R-R state would be $F^{\alpha \beta}$ with the equations of motion $\partial^{\alpha \gamma} F^{\gamma \beta}=\partial^{\beta \gamma} F^{\alpha \gamma}=0$. However, in this case, there is no action in terms of just $F^{\alpha \beta}$ (i.e., without gauge fields) which can reproduce these equations of motion. This is especially problematic for the type IIB superstring where one needs an infinite number of auxiliary fields to write an action for the self-dual five-form field strength $[4,11]$.
Superstring field theory (the massless NS-NS, NS-R, and R-NS sectors). - The NS-NS, NS-R, and R-NS sectors of the type II superstring are similarly described by a string field constructed from non-negative modes acting on a vacuum. The vacuum $|0\rangle_{\mathrm{NS}-\mathrm{NS}}$ will be defined in the picture $\left(P_{L}, P_{R}\right)=(-1,-1),|0\rangle_{\mathrm{NS}-\mathrm{R}}^{\alpha}$ will be defined in the picture $\left(P_{L}, P_{R}\right)=\left(-1,-\frac{1}{2}\right)$ and $|0\rangle_{\mathrm{R}-\mathrm{NS}}^{\alpha}$ will be defined in the picture $\left(P_{L}, P_{R}\right)=\left(-\frac{3}{2},-1\right)$. So like $|0\rangle_{\mathrm{R}-\mathrm{R}}^{\alpha \beta}$, these vacua are annihilated by all negative modes and by $\beta_{R}^{0}, \gamma_{L}^{0}$, and $b_{L / R}^{0}$. In terms of the SL(2)-invariant vacuum, these vacua are $c_{L} e^{-\phi_{L}} c_{R} e^{-\phi_{R}}, c_{L} e^{-\phi_{L}} c_{R} e^{-\frac{1}{2} \phi_{R}}$, and $c_{L} e^{-\frac{3}{2} \phi_{L}} c_{R} e^{-\phi_{R}}$.

The massless states in these sectors are described by

$$
\begin{aligned}
|\Phi\rangle_{\mathrm{NS}-\mathrm{NS}}= & \left\{\left[g_{\mu \nu}(x)+b_{\mu \nu}(x)\right] \psi_{L}^{\frac{1}{2} \mu} \psi_{R}^{\frac{1}{2} \nu}+\phi(x) \beta_{L}^{\frac{1}{2}} \gamma_{R}^{\frac{1}{2}}+\tilde{\phi}(x) \gamma_{L}^{\frac{1}{2}} \beta_{R}^{\frac{1}{2}}+B_{\mu}(x) c_{+}^{0} \beta_{L}^{\frac{1}{2}} \psi_{R}^{\frac{1}{2} \mu}+\tilde{B}_{\mu}(x) c_{+}^{0} \psi_{L}^{\frac{1}{2} \mu} \beta_{R}^{\frac{1}{2}}\right\} \\
& \times|0\rangle_{\mathrm{NS}-\mathrm{NS}} \\
|\Phi\rangle_{\mathrm{NS}-\mathrm{R}}= & {\left[\chi_{\mu}^{\alpha}(x) \psi_{L}^{\frac{1}{2} \mu}+\tau^{\alpha}(x) c_{+}^{0} \beta_{L}^{\frac{1}{2}}+\Xi^{\alpha}(x) \beta_{L}^{\frac{1}{2}} \gamma_{R}^{0}\right]|0\rangle_{\mathrm{NS}-\mathrm{R}}^{\alpha} } \\
|\Phi\rangle_{\mathrm{R}-\mathrm{NS}}= & {\left[\tilde{\chi}_{\mu}^{\alpha}(x) c_{+}^{0} \beta_{L}^{0} \psi_{R}^{\frac{1}{2} \mu}+\tilde{r}^{\alpha}(x) c_{+}^{0} \beta_{R}^{\frac{1}{2}}+\tilde{\Xi}^{\alpha}(x) c_{+}^{0}\left(\beta_{L}^{0}\right)^{2} \gamma_{R}^{\frac{1}{2}}+M_{\mu}^{\alpha}(x) \psi_{R}^{\frac{1}{2} \mu}+N^{\alpha}(x) \beta_{L}^{0} \gamma_{R}^{\frac{1}{2}}\right]|0\rangle_{\mathrm{R}-\mathrm{NS}}^{\alpha} . }
\end{aligned}
$$

Note that $\left[B^{\mu}, \tilde{B}^{\mu}, \tau^{\alpha}, \tilde{\tau}^{\alpha}\right]$ are auxiliary fields, and $[\tilde{\phi}$, $\left.M_{\mu}^{\alpha}, N^{\alpha}\right]$ can be eliminated by algebraic gauge transformations.

The remaining fields transform under coordinate reparametrizations, $b_{\mu \nu}$ gauge transformations, and $N=$ 2 local supersymmetry transformations as

$$
\begin{aligned}
\delta\left(g_{\mu \nu}+b_{\mu \nu}\right) & =\partial_{\mu} y_{\nu}+\partial_{\nu} \tilde{y}_{\mu}, \\
\delta \phi & =\partial^{\mu}\left(y_{\mu}+\tilde{y}_{\mu}\right), \\
\delta \chi_{\mu}^{\alpha} & =\partial_{\mu} \epsilon^{\alpha}, \quad \delta \tilde{\chi}_{\mu}^{\alpha}=\partial_{\mu} \tilde{\epsilon}^{\alpha}, \\
\delta \Xi^{\alpha} & =\partial^{\alpha \beta} \epsilon^{\beta}, \quad \delta \tilde{\Xi}^{\alpha}=\partial^{\alpha \beta} \tilde{\epsilon}^{\beta},
\end{aligned}
$$

which are parametrized by the gauge field

$$
\begin{aligned}
|\Lambda\rangle_{\mathrm{NS}-\mathrm{NS}}= & {\left[y_{\mu}(x) \beta_{L}^{\frac{1}{2}} \psi_{L}^{\frac{1}{2} \mu}+\tilde{y}_{\mu}(x) \psi_{L}^{\frac{1}{2} \mu} \beta_{R}^{\frac{1}{2}}\right.} \\
& \left.\quad-\partial^{\mu} \tilde{y}_{\mu} c_{+}^{0} \beta_{L}^{\frac{1}{2}} \beta_{R}^{\frac{1}{2}}\right]|0\rangle_{\mathrm{NS}-\mathrm{NS}} \\
|\Lambda\rangle_{\mathrm{NS}-\mathrm{R}}= & \epsilon^{\alpha}(x) \beta_{L}^{\frac{1}{2}}|0\rangle_{\mathrm{NS}-\mathrm{R}}^{\alpha} \\
|\Lambda\rangle_{\mathrm{R}-\mathrm{NS}}^{\alpha}= & \tilde{\epsilon}^{\alpha}(x)\left[c_{+}^{0} \beta_{L}^{0} \beta_{R}^{\frac{1}{2}}-c_{+}^{0}\left(\beta_{L}^{0}\right)^{3} \gamma_{R}^{\frac{1}{2}}\right]|0\rangle_{\mathrm{R}-\mathrm{NS}}^{\alpha} .
\end{aligned}
$$

Note that these transformations have been chosen to preserve the gauge $\tilde{\phi}=M_{\mu}^{\alpha}=N^{\alpha}=0$.

Conserved charges. - When the gauge parameters $\left[y_{\mu}, \tilde{y}_{\mu}, \boldsymbol{\epsilon}^{\alpha}, \tilde{\boldsymbol{\epsilon}}^{\alpha}, \boldsymbol{\rho}_{(0)}^{\alpha \beta}, \rho_{(1)}^{\alpha \beta}\right]$ of Eqs. (10) and (4) are constants $Q|\Lambda\rangle=0$. The -1 ghost-number states described by $|\Lambda\rangle$ are

$$
\begin{aligned}
P_{R}^{\mu} & =\beta_{L}^{\frac{1}{2}} \psi_{R}^{\frac{1}{2} \mu}|0\rangle_{\mathrm{NS}-\mathrm{NS}}, \quad P_{L}^{\mu}=\psi_{L}^{\frac{1}{2} \mu} \beta_{R}^{\frac{1}{2}}|0\rangle_{\mathrm{NS}-\mathrm{NS}}, \\
q_{R}^{\alpha} & =\beta_{L}^{\frac{1}{2}}|0\rangle_{\mathrm{NS}-\mathrm{R}}^{\alpha}, \\
q_{L}^{\alpha} & =\left[c_{+}^{0} \beta_{L}^{0} \beta_{R}^{\frac{1}{2}}-c_{+}^{0}\left(\beta_{L}^{0}\right)^{3} \gamma_{R}^{\frac{1}{2}}\right]|0\rangle_{\mathrm{R}-\mathrm{NS}}^{\alpha}, \\
C_{(0)}^{\alpha \beta} & =\beta_{L}^{0}|0\rangle_{\mathrm{R}-\mathrm{R}}^{\alpha \beta}, \quad C_{(1)}^{\alpha \beta}=\left(\beta_{L}^{0}\right)^{2} \gamma_{R}^{0}|0\rangle_{\mathrm{R}-\mathrm{R}}^{\alpha \beta},
\end{aligned}
$$

which are the conserved charges associated with the global part of the gauge transformations. These states cannot be written as $Q|\Omega\rangle$, where $|\Omega\rangle$ has a finite number of terms, so they are in the BRST cohomology at -1 ghost number.

Note that the conserved R-R charges $C_{(0)}^{\alpha \beta}$ and $C_{(1)}^{\alpha \beta}$ are in the same cohomology class as the states $\left(\beta_{L}^{0}\right)^{n} \times$ $\left(\gamma_{R}^{0}\right)^{n-1}|0\rangle_{\mathrm{R}-\mathrm{R}}^{\alpha \beta}$, where $n$ is an arbitrarily large odd or even number. This means that the R-R charges act as BRST-trivial operators on any superstring state which carries a finite left-moving ghost number. This includes all perturbative superstring states, but not $D$-brane boundary states which contain the ghost dependence $e^{\beta_{L}^{0} \gamma_{R}^{0}}$ [12]. For this reason, $D$-branes can carry a nonzero R-R charge but perturbative superstring states cannot. Since R-R charge is related to momentum in the eleventh direction, there is a relation between nontrivial dependence on world sheet ghosts and nontrivial dependence on the eleventh direction.

By hitting with the left- and right-moving picturechanging operators $Z_{L} Z_{R}$, it is easy to see that the NS - NS charges are the $\left(P_{L}, P_{R}\right)=(-1,-1)$ versions of the translation and NS-NS one-brane generators $\int d \sigma\left(\partial_{\tau} x^{\mu} \pm \partial_{\sigma} x^{\mu}\right)$, and the NS-R and R-NS charges are the $\left(-\frac{3}{2},-1\right)$ and $(-1,-1 / 2)$ versions of the $N=2$ supersymmetry generators $q_{L / R}^{\alpha}=\int d \sigma e^{-\frac{1}{2} \phi_{L / R}} \Sigma_{L / R}^{\alpha}$. However, $Z_{L} Z_{R}$ annihilates the R-R charges so one needs to develop a "picture-raising" prescription which preserves BRST cohomology. This has been accomplished in [13] where it will be shown that the R-R charge $C_{(0)}^{\alpha \beta}$ of (11) is the $\left(P_{L}, P_{R}\right)=\left(-\frac{3}{2},-\frac{1}{2}\right)$ version of the $\mathrm{R}-\mathrm{R}$ charge $\int d \sigma \lambda_{L}^{\alpha} \lambda_{R}^{\beta}$ which was proposed in Ref. [14] using a twistorlike construction.

Supersymmetry algebra of the superstring. - Finally, it will be shown that the supersymmetry transformations of the superstring close to an $N=2$ SUSY algebra including R-R central charges. Since the SUSY generators carry a picture, one needs to be careful to choose the right picture 
when defining the super-symmetry transformations of the string field. For a global supersymmetry transformation parameterized by $u_{\alpha}$ and $\tilde{u}_{\alpha}$, the correct choice is

$$
\begin{aligned}
\delta|\Phi\rangle_{\mathrm{NS}-\mathrm{NS}} & =\tilde{u}^{\alpha} \bar{q}_{L}^{\alpha}|\Phi\rangle_{\mathrm{R}-\mathrm{NS}}+u^{\alpha} q_{R}^{\alpha}|\Phi\rangle_{\mathrm{NS}-\mathrm{R}}, \\
\delta|\Phi\rangle_{\mathrm{R}-\mathrm{NS}} & =\tilde{u}^{\alpha} q_{L}^{\alpha}|\Phi\rangle_{\mathrm{NS}-\mathrm{NS}}+u^{\alpha} q_{R}^{\alpha}|\Phi\rangle_{\mathrm{R}-\mathrm{R}}, \\
\delta|\Phi\rangle_{\mathrm{NS}-\mathrm{R}} & =\tilde{u}^{\alpha} \bar{q}_{L}^{\alpha}|\Phi\rangle_{\mathrm{R}-\mathrm{R}}+u^{\alpha} \bar{q}_{L}^{\alpha}|\Phi\rangle_{\mathrm{NS}-\mathrm{NS}}, \\
\delta|\Phi\rangle_{\mathrm{R}-\mathrm{R}} & =\tilde{u}^{\alpha} q_{L}^{\alpha}|\Phi\rangle_{\mathrm{NS}-\mathrm{R}}+u^{\alpha} \bar{q}_{L}^{\alpha}|\Phi\rangle_{\mathrm{R}-\mathrm{NS}},
\end{aligned}
$$

where $q_{L / R}^{\alpha}$ carries a $-\frac{1}{2}$ picture and $\bar{q}_{L / R}^{\alpha}$ carries a $+\frac{1}{2}$ picture. The easiest way to define the action of $q_{L / R}^{\alpha}$ or $\bar{q}_{L / R}^{\alpha}$ on the string field is to first write the string field in terms of the SL(2)-invariant vacuum, then take the contour integral of $\int d \sigma e^{-\frac{1}{2} \phi_{L / R}} \Sigma_{L / R}^{\alpha}$ or $\int d \sigma\left(e^{\frac{1}{2} \phi_{L / R}} \Gamma_{\mu}^{\alpha \beta} \Sigma_{L / R}^{\beta} \partial_{L / R} x^{\mu}+b \eta e^{\frac{3}{2} \phi_{L / R}} \Sigma_{L / R}^{\alpha}\right)$ around the vertex operator, and finally reexpress the resulting vertex operator in terms of the string vacuum. Note that in the gauge where $\tilde{\phi}=M_{\mu}^{\alpha}=N^{\alpha}=A_{(2)}^{\alpha \beta}=0$, one also needs to include compensating gauge transformations which cancel the ${ }_{\alpha}$ supersymmetry transformation of $\tilde{\phi}$, $M_{\mu}^{\alpha}, N^{\alpha}$, and $A_{(2)}^{\alpha \beta}$. For example, $\delta|\Phi\rangle_{\text {NS-NS needs to }}$ include the term $Q \tilde{u}^{\alpha} \tilde{\tau}^{\alpha}(x) c_{+}^{0} \beta_{L}^{\frac{1}{2}} \beta_{R}^{\frac{1}{2}}|0\rangle_{\mathrm{NS}-\mathrm{NS}}$ to cancel the variation of $\tilde{\phi}$, and $\delta|\Phi\rangle_{\mathrm{R}-\mathrm{R}}$ needs to include the term $Q u^{\beta} \tilde{\Xi}^{\alpha}(x) c_{+}^{0}\left(\beta_{L}^{0}\right)^{2}|0\rangle_{\mathrm{R}-\mathrm{R}}^{\alpha \beta}$ to cancel the variation of $A_{(2)}^{\alpha \beta}$.

The simplest nontrivial computation is the fieldindependent part of the transformation resulting from the commutator of a global supersymmetry transformation with a local supersymmetry transformation. (The field-independent part vanishes for the commutator of two global SUSY transformations, and is complicated for the commutator of two local SUSY transformations.)

Using (12) and (10), the field-independent part of this commutator is

$$
\begin{aligned}
\delta|\Phi\rangle_{\mathrm{NS}-\mathrm{NS}} & =\tilde{u}^{\alpha} \bar{q}_{L}^{\alpha} Q \tilde{\epsilon}^{\beta} c_{+}^{0}\left[\beta_{L}^{0} \beta_{R}^{\frac{1}{2}}-\left(\beta_{L}^{0}\right)^{3} \psi_{R}^{\frac{1}{2} \mu}\right]|0\rangle_{\mathrm{R}-\mathrm{NS}}^{\beta}+u^{\alpha} q_{R}^{\alpha} Q \epsilon^{\beta} \beta_{L}^{\frac{1}{2}}|0\rangle_{\mathrm{NS}-\mathrm{R}}^{\beta}+Q \tilde{u}^{\alpha} \partial^{\alpha \gamma} \tilde{\epsilon}^{\gamma} c_{+}^{0} \beta_{L}^{\frac{1}{2}} \beta_{R}^{\frac{1}{2}}|0\rangle_{\mathrm{NS}-\mathrm{NS}}, \\
\delta|\Phi\rangle_{\mathrm{NS}-\mathrm{R}} & =\delta|\Phi\rangle_{\mathrm{R}-\mathrm{NS}}=0, \\
\delta|\Phi\rangle_{\mathrm{R}-\mathrm{R}} & =\tilde{u}^{\alpha} q_{L}^{\alpha} Q \epsilon^{\beta} \beta_{L}^{\frac{1}{2}}|0\rangle_{\mathrm{NS}-\mathrm{R}}^{\beta}+u^{\alpha} \bar{q}_{R}^{\alpha} Q \tilde{\epsilon}^{\beta} c_{+}^{0}\left[\beta_{L}^{0} \beta_{R}^{\frac{1}{2}}-\left(\beta_{L}^{0}\right)^{3} \gamma_{L}^{\frac{1}{2}}\right]|0\rangle_{\mathrm{R}-\mathrm{NS}}^{\beta}+Q u^{\beta} \partial^{\alpha \gamma} \tilde{\boldsymbol{\epsilon}}^{\gamma} c_{+}^{0}\left(\beta_{L}^{0}\right)^{2}|0\rangle_{\mathrm{R}-\mathrm{R}}^{\alpha \beta},
\end{aligned}
$$

where $\left[u^{\alpha}, \tilde{u}^{\alpha}\right]$ are global parameters and $\left[\epsilon^{\alpha}(x), \tilde{\epsilon}^{\alpha}(x)\right]$ are local parameters.

Converting to the SL(2)-invariant vacuum to compute the action of $q_{L / R}^{\alpha}$ and $\bar{q}_{L / R}^{\alpha}$, and then reexpressing in terms of the original vacuum, one finds

$$
\begin{aligned}
\delta|\Phi\rangle_{\mathrm{NS}-\mathrm{NS}} & =\Gamma_{\alpha \beta}^{\mu} Q\left(u^{\alpha} \epsilon^{\beta} \beta_{L}^{\frac{1}{2}} \psi_{R}^{\frac{1}{2} \mu}+\tilde{u}^{\alpha} \tilde{\boldsymbol{\epsilon}}^{\beta} \psi_{R}^{\frac{1}{2} \mu} \beta_{R}^{\frac{1}{2}}+\tilde{u}^{\alpha} \partial^{\beta \gamma} \tilde{\epsilon}^{\gamma} c_{+}^{0} \beta_{L}^{\frac{1}{2}} \beta_{R}^{\frac{1}{2}}\right)|0\rangle_{\mathrm{NS}-\mathrm{NS}}, \\
\delta|\Phi\rangle_{\mathrm{NS}-\mathrm{R}} & =\delta|\Phi\rangle_{\mathrm{R}-\mathrm{NS}}=0 \\
\delta|\Phi\rangle_{\mathrm{R}-\mathrm{R}} & =Q\left(\tilde{u}^{\alpha} \epsilon^{\beta}+\mu^{\beta} \tilde{\boldsymbol{\epsilon}}^{\alpha}\right) \beta_{L}^{0}|0\rangle_{\mathrm{R}-\mathrm{R}}^{\alpha \beta} .
\end{aligned}
$$

So the field-independent part of the transformation resulting form the commutator of a local and global supersymmetry transformation is given by

$$
\begin{aligned}
{\left[\delta_{q_{L}^{\alpha}}\left(\tilde{\epsilon}^{\alpha}(x)\right)+\delta_{q_{R}^{\alpha}}\left(\epsilon^{\alpha}(x)\right), \delta_{q_{L}^{\beta}}\left(\tilde{u}^{\beta}\right)+\delta_{q_{R}^{\beta}}\left(\tilde{u}^{\beta}\right)\right]=} & \delta_{P_{R}^{\mu}}\left(\epsilon^{\alpha}(x) \Gamma_{\alpha \beta}^{\mu} u^{\beta}+\delta_{P_{L}^{\mu}}\left(\tilde{\epsilon}^{\alpha}(x) \Gamma_{\alpha \beta}^{\mu} \tilde{u}^{\beta}\right)\right. \\
& +\delta_{C_{(0)}^{\alpha \beta}}\left(\tilde{u}^{\alpha} \epsilon^{\beta}(x)+u^{\beta} \tilde{\epsilon}^{\alpha}(x)\right),
\end{aligned}
$$

where $\delta_{P_{R}^{\mu}}\left(y^{\mu}\right)$ and $\delta_{P_{L}^{\mu}}\left(\tilde{y}^{\mu}\right)$ are NS-NS gauge transformations parametrized by $y^{\mu}$ and $\tilde{y}^{\mu}$ of Eq. (10), $\delta_{q_{R}^{\alpha}}\left(\epsilon^{\alpha}\right)$ and $\delta_{q_{L}^{\alpha}}\left(\tilde{\boldsymbol{\epsilon}}^{\alpha}\right)$ are R-NS and NS-R gauge transformations parametrized by $\epsilon^{\alpha}$ and $\tilde{\epsilon}^{\alpha}$ of Eq. (10), and $\delta_{C_{(0)}^{\alpha \beta}}\left(_{\alpha \beta}^{\alpha \beta}\right)$ are R-R gauge transformations parametrized by $\rho_{(0)}^{\alpha \beta}$ of Eq. (4). Therefore, supersymmetry transformations of the type II superstring form an $N=2$ SUSY algebra with an NS-NS one-brane central charge and with $256 \mathrm{R}-\mathrm{R}$ central charges.

I thank I. Bars, S. Ramgoolam, and W. Siegel for useful conversations. This work was financially supported by FAPESP Grant No. 96/05524-0.

*Electronic address: nberkovi@ift.unesp.br

[1] J. Polchinski, Phys. Rev. Lett. 75, 4724 (1995).

[2] D. Polyakov, hep-th/9704191.

[3] J.A. Azcarraga, J.P. Gauntlett, J.M. Izquierdo, and P. K. Townsend, Phys. Rev. Lett. 63, 2443 (1989).
[4] N. Berkovits, Phys. Lett. B 388, 743 (1996); N. Berkovits, Phys. Lett. B 395, 28 (1997).

[5] E.S. Fradkin and A. A. Tseytlin, Phys. Lett. 158B, 316 (1985).

[6] N. Berkovits and W. Siegel, Nucl. Phys. B462, 213 (1996).

[7] B. deWit, P. G. Lauwers, and A. Van Proeyen, Nucl. Phys. B255, 569 (1985).

[8] W. Siegel, Int. J. Mod. Phys. A 6, 3997 (1991); N. Berkovits, M. T. Hatsuda, and W. Siegel, Nucl. Phys. B371, 434 (1991).

[9] M. Bianchi, G. Pradisi, and A. Sagnotti, Nucl. Phys. B376, 365 (1992).

[10] S. Ramgoolam (private communication).

[11] B. McClain, Y.S. Wu, and F. Yu, Nucl. Phys. B343, 689 (1990); C. Wotzasek, Phys. Rev. Lett. 66, 129 (1991); I. Bengtsson and A. Kleppe, hep-th/9609102.

[12] S. A. Yost, Nucl. Phys. B321, 629 (1989).

[13] N. Berkovits, (to be published).

[14] N. Berkovits, e-print IFT-P.033/97, hep-th/9704109. 